Ann. Biol. anim. Bioch. Biophys., I976, 16 (I), I3-24.

\title{
ANALYSE DES PROTIDES DES GANGUES OVULAIRES DE PLEURODELES WALTLII MICHAH (AMPHIBIEN, URODÈLE)
}

\author{
P. JEGO \\ Laboratoire de Biologie de la Reproduction, \\ Groupe de Recherches en Biologie générale et cellulaire (E. R. A. 129), \\ $U E R$, Sciences biologiques, \\ Avenue du Général Leclerc, \\ 35031 Rennes
}

\section{RÉSUMÉ}

Quatre fractions (FI, FM, FEi et FEe) ont été préparées, après isolement mécanique, à partir des gangues ovulaires de Pleurodeles waltlii міснан.

Leur analyse révèle que si elles sont toutes très riches en sérine et thréonine, elles sont constituées d'éléments protidiques nettement différents; ces fractions peuvent cependant se classer en deux catégories:

I $^{0}$ FI et FEi (correspondant à la gangue interne et à la partie interne de la gangue externe) ont des constituants sulfatés à poids moléculaire inférieur ou égal à Ioo ooo daltons solubles dans l'eau ou en milieu faiblement alcalin et particulièrement riches en sérine et en thréonine.

$2^{\circ} \mathrm{FM}$ et FEe (correspondant à la gangue moyenne et à la partie externe de la gangue externe) ont des constituants très peu sulfatés à poids moléculaire supérieur ou nettement supérieur à Ioo ooo daltons, nettement insolubles même en milieu faiblement alcalin, riches en acides glutamique et aspartique et en acides aminés à chaîne latérale hydrophobe.

\section{INTRODUCTION}

Les œufs d'amphibiens sont recouverts de plusieurs gangues d'aspect gélatineux qui assurent leur protection puis celle de l'embryon. Le rôle de ces gangues, ne se limite pas à celui d'une barrière physique vis-à-vis des agresseurs éventuels de l'œuf puisqu'elles exercent un effet "capacitant " sur les spermatozoïdes. De très nombreuses expériences montrent, en effet, que la proportion d'ovocytes fécondés est beaucoup plus grande lorsqu'ils sont munis de leurs gangues que lorsqu'ils sont nus. (Batarlion, I9I9; Good et Daniel, I943; Kambara, I953; TChou-Su et Wang, 
I956 ; Aplington, I957 ; Hughes, I957 ; Nadamitsu, I957 ; Shaver et Barch, I960; Glick et SHAVER, I963; ElLINSON, I97I). La fécondation d'ovocytes dégangués est possible si 1'on utilise des spermatozoïdes préalablement " capacités " par un séjour au contact de gangues ovulaires. (SUBTELNY et BRADT, I96r ; KATAGIRI, I966 ; BARbIERI et RAISMAN, I969 ; SHIVERs et JAMES, I970 ; WolF et HEDRICK, I97I). Des facteurs capacitants ont même pu être isolé chez certains anoures : Bufo Arenarum (BARBIERI et OtERINo, I972) ; Bufo Bufo (KATAGIRI, I973) et Xenopus Laevis (Oliphan'T et HEDRICK, I97I).

Cet aspect important de la fécondation, partiellement connu chez les Anoures, mérite d'être étudié chez les Urodèles. La recherche du (des) facteur (s) capacitant éventuel nécessite une bonne connaissance de la composition chimique des gangues. Ce travail axé sur les composants protidiques ainsi que le précédent se rapportant aux composants glucidiques des gangues (JEGO, r974) permettra d'effectuer cette recherche dans de meilleures conditions.

Il faut noter par ailleurs que ces gangues sont totalement sécrétées par l'oviducte qui s'est révélé être un excellent organe cible pour l'œstradiol (BoISSEAU, I974). La détermination de la composition chimique de celles-ci constitue un apport essentiel à l'étude des mécanismes d'action de l'œestradiol sur l'oviducte déjà entreprise (JEGo et al., 1974).

Comme le révèle l'histochimie (BoISSEAu et al., I974) ainsi que la répartition des glucides (JEGo, I974), le matériel entourant l'œuf est constitué du produit de sécrétion de régions distinctes de l'oviducte. Il se répartit en plusieurs couches successives que nous avons fractionnées de façon à les étudier séparément.

\section{MATÉRIEL ETT MÉTHODES}

\section{I. - Séparation des fractions}

Une analyse histochimique préalable nous a permis de mettre en évidence que l'ouf de Pleurodeles waltlii мгснан est entouré de cinq enveloppes concentriques appelées respectivement gangue interne (GI), gangue moyenne (GM) et gangue externe (GE), la dernière étant subdivisée en trois couches $\mathrm{GE}_{1}, \mathrm{GE}_{2}$ et $\mathrm{GE}_{3}$, (BoIsseAU et al., I974). Les prélèvements sont effectués entre I 2 et 24 heures après la ponte des oeufs; les gangues ovulaires sont fractionnées mécaniquement comme il est indiqué dans la figure $I$.

\section{2. - Électrophorèse sur gel de polyacrylamide}

Après complexation par le SDS (sodium dodecyl sulfate), les quatre fractions sont soumises à une électrophorèse dans un système discontinu où la migration des composés est fonction de leur poids moléculaire (NEVILLE, I97I).

Les tampons utilisés ont les compositions suivantes :

- cuve supérieure ( $\mathrm{pH}: 8,64)$ acide borique $0,04 \mathrm{M}$, tris 0,04IM et SDS o, I p. Ioo ;

- gel supérieur $(\mathrm{pH}: 6, \mathbf{I}) \mathrm{H}_{2} \mathrm{SO}_{4}: 0,0,0267 \mathrm{M}$ et tris $0,054 \mathrm{IM}$;

- gel inférieur et cuve inférieure $(\mathrm{pH}: 9,8 \mathrm{I}): \mathrm{HCl}: 0,0237 \mathrm{M}$ et tris $1,3{ }_{4} 7 \mathrm{M}$.

Le gel supérieur contient 3 p. Ioo d'acrylamide et 0,2 p. Ioo de bisacrylamide $(3,2 \times 6,25$ d'après les notations de HJERTEN, I962); le gel inférieur contient I I p. roo d'acrylamide et $0, \mathrm{I}$ p. roo de bisacrylamide $(\mathrm{I}, \mathrm{I} \times 0,9)$. La polymérisation est obtenue dans les deux cas avec 0, I 5 p. Ioo de $\mathrm{N}, \mathrm{N}, \mathrm{N}, \mathrm{N}$-tétraméthyléthylènediamine et o,05 p. Ioo de persulfate d'ammonium.

Les différentes fractions sont mises en suspension dans une solution de $\mathrm{Na}_{2} \mathrm{CO}_{3} 0,05 \mathrm{M}$ dans laquelle est ajouté le SDS; après I minute d’action du SDS, du mercaptoéthanol est introduit 
dans le milieu (Io p. Ioo v/v). Le mélange est finalement mis à dialyser pendant une nuit contre le tampon du gel supérieur contenant o, I p. Ioo de SDS, 0,05 p. Ioo de dithiothreitol, 2 p. Ioo de saccharose et une trace de bleu de bromophénol.
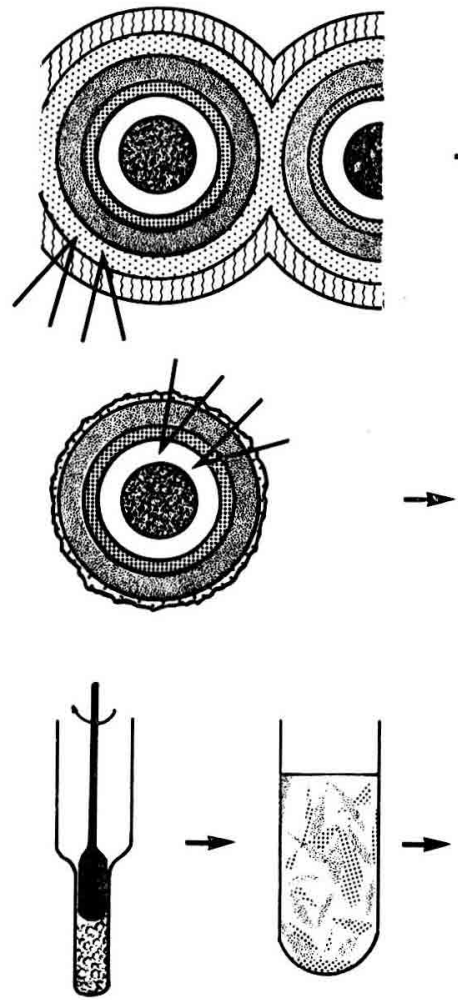
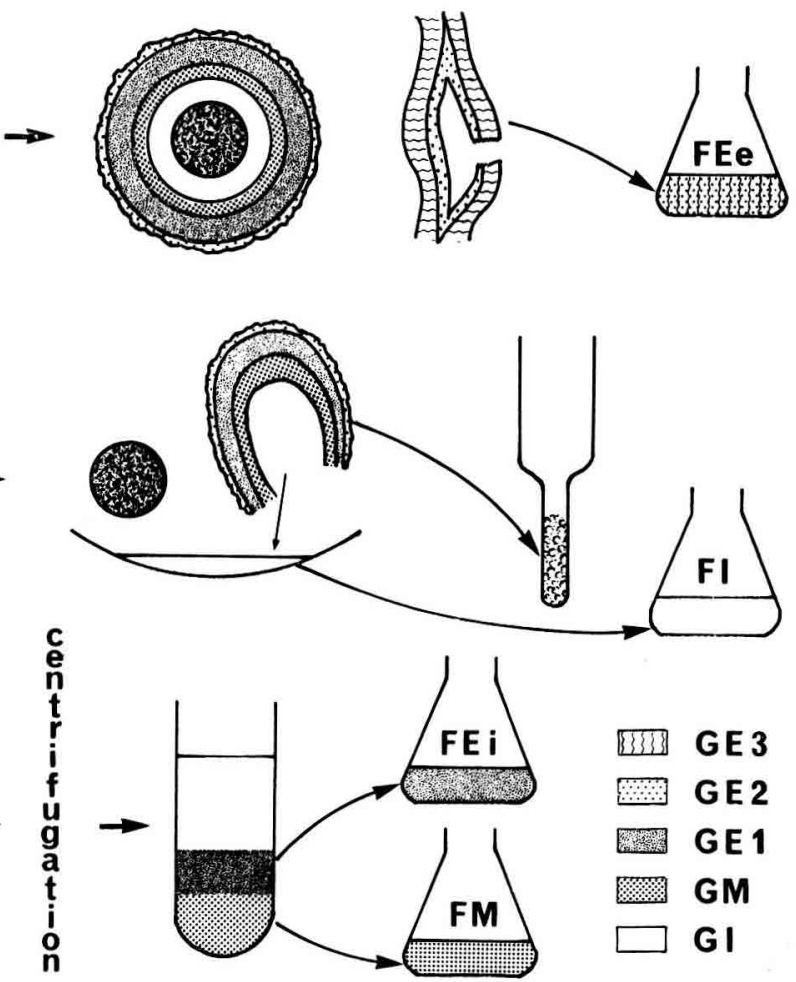

FIG. I. - Schéma indiquant les différentes étapes du fractionnement des gangues ovulaires

$\mathrm{GI}, \mathrm{GM}$ et $\mathrm{GE}$ : gangues interne, moyenne et externe; $\mathrm{GE}_{1}, \mathrm{GE}_{2}$ et $\mathrm{GE}_{3}$ correspondent aux trois couches de la gangue externe. FI, FM, FEi et FEe : fractions isolées à partir des gangues.

Partie supérieure du schéma: Séparation à la pince de FEe.

Partie moyenne: Après ouverture des gangues débarrassées de FEe on recueille FI qui est liquide; les gangues restantes (privées de FEe et FI) sont lavées dans l'eau distillée.

Partie inférieure : Après broyage dans un potter teflon-verre (5 à ro aller-retours) ces gangues restantes sont centrifugées à 20 ooo $g$ pendant $\mathrm{I}$ heure. Le culot dense est appelé FM et la phase intermédiaire FEi. Le surnageant (eau de rinçage) ne contient pratiquement rien.

Avant leur utilisation, les quatre fractions sont lyophilisées. (Un contrôle histochimique a permis de comparer des frottis de fractions à des coupes de gangues in situ: FI et FM correspondent respectivement à GI et GM. FEi et FEe contiennent respectivement $G_{1}$ et $G_{3}$. $G_{2}$ se répartit entre FEi et $\mathrm{FEe}$ avec une nette prépondérance pour $\mathrm{FEe})$.

Au sommet de chaque tube 5 à $200 \mu g$ de protéines sont déposés. L'intensité utilisée est de I,5 $\mathrm{mA}$ par tube, le pôle positif du générateur étant relié à la cuve inférieure de l'appareil à électrophorèse. Le front de migration est repéré grâce au bleu de bromophénol. Après précipitation par l'acide trichloracétique à 5 p. Ioo, la coloration est effectuée par le bleu de Coomassie à o, I25 p. Ioo dans l'acide acétique 7,5 p. I 00 ou par l'amido-schwartz à o,6 p. roo dans un mélange d'eau, de méthanol et d'acide acétique $(9: 9: 2)$.

La détermination de la relation existant entre le poids moléculaire et le $\mathrm{Rf}$ a été réalisée en utilisant des protéines témoins. Nous avons ainsi établi une courbe indiquant les variations du logarithme de Rf en fonction du poids moléculaire; cette courbe (il s'agit d'une droite dont la pente s'infléchit pour les poids moléculaires élevés) correspondant exactement à celle obtenue par Neville (r97I). 


\section{3. - Dosage des Protéines}

Le dosage des protéines a été réalisé selon la technique de Lowny appliquée soit aux protéines solubles soit aux protéines insolubles (LOWRY et al., 195I).

$$
\text { 4. - Dosage des acides aminés }
$$

a) Hydrolyse des fractions:

L'hydrolyse est réalisée, en tubes scellés, par l'acide chlorydrique $6 \mathrm{~N}$ à ro $5^{\circ} \mathrm{C}$. A partir de chaque fraction, trois temps d'hydrolyse durant respectivement $24,4^{8}$ et 72 heures ont été effectués.

\section{b) Technique de dosage:}

Après élimination de l'acide chlorhydrique (évaporation sous vide), les échantillons sont analysés au moyen d'un analyseur automatique d'acides aminés Technicon (Résine $C_{2}$ ) élution par gradient de $\mathrm{pH}$ (tampons citrate de sodium) ( $\mathrm{pH}: 2,75$ à 6, Io), coloration par la ninhydrine. Avec cette méthode, il arrive parfois que la cystéine et la méthionine interfèrent partiellement avec les osamines (glucosamine et galactosamine). Cette difficulté a pu être surmontée en analysant parallèlement, chaque fois, par cette méthode, les acides aminés neutres séparés des autres groupes d'acides aminés et des osamines par HV électrophorèse (effectuée dans un tampon $\mathrm{pH}: 5,3$ composé. d'une solution aqueuse de pyridine ( $\mathrm{r}$ p. Ioo) et d'acide acétique (o,4 P. I00), (2 900 V, 30 minutes)

Les élutions ont été réalisées par l'acide chlorhydrique $\frac{\mathrm{N}}{\mathrm{I} \mathrm{Ooo}}$ pendant ${ }_{4} 8$ heures.

$$
\text { 5. - Dosage des ions sulfates }
$$

Les ions sulfates ont été dosés selon la technique de Dogson et Price (1962) modifiée par PICARD et al. (1973). Après hydrolyse des fractions par l'acide chlorhydrique ${ }_{4} \mathrm{~N}$ pendant 3 heures à $100^{\circ} \mathrm{C}$, les sulfates sont précipités par un réactif contenant $80 \mathrm{ml}$ de chlorure de baryum à Io $\mathrm{p}$. Ioo dans l'eau bidistillée et $\mathrm{r} 5 \mathrm{ml}$ de tween 80 puis dosés en milieu $\mathrm{HCl} \mathrm{N}$ par néphélométrie à $500 \mathrm{~nm}$.

\section{RÉSULTATS}

L'observation de la planche I permet de classer les fractions en deux catégories selon l'ordre de grandeur du poids moléculaire de leurs constituants.

Les constituants des fractions FI et FEi ont des poids moléculaires variant entre Io 000 et roo ooo daltons alors que ceux des deux autres fractions ont des poids moléculaires supérieurs ou nettement supérieurs à roo ooo daltons.

Quatre composés ayant des poids moléculaires respectivement voisins de I5 000, 28000,35000 et 48000 daltons sont communs à $\mathrm{FI}$ et $\mathrm{FEi} ; \mathrm{FEi}$ possède en outre un constituant de poids moléculaire voisin de 90000 daltons. La fraction moyenne possède deux groupes de constituants : un groupe de trois constituants à poids moléculaires variant entre 200000 et 350000 daltons et un groupe de deux constituants à poids moléculaires beaucoup plus élevés. La fraction la plus externe (FEe) contient plusieurs composés à poids moléculaires encore plus élevés que ceux de la fraction moyenne.

Plusieurs composés sont communs à plusieurs fractions; ceci provient d'une contamination des fractions entre elles intervenant lors de leur isolement ou peutêtre se produisant naturellement in situ (voir discussion). 
Pour les fractions FM et FEe, une grande quantité de protéines est restée dans le gel de concentration ; ceci est dû à une solubilisation incomplète des produits par le traitement SDS + Mercaptoéthanol. En effet, si on effectue la même expérience avec des fractions FM et FEe préalablement solubilisées par 1'urée $8 \mathrm{M}$ contenant Io p. Ioo de mercaptoéthanol (traitement suivi par une dialyse qui élimine les solvants), toutes les protéines pénètrent dans le gel de diffusion. Flles migrent d'ailleurs de la même manière qu'après un traitement simple par le SDS + Mercaptoéthanol. Pour la détermination des poids moléculaires, nous avons préféré n'utiliser que le traitement préconisé par NEVII,LE qui est beaucoup moins dénaturant et permet néanmoins de mettre en évidence toutes les catégories de protéines.

\section{Teneur en protéines}

Les résultats concernant les teneurs en protéines des différentes fractions présentées dans le tableau I n'ont qu'une valeur indicative puisque la référence utilisée a toujours été la sérum albumine alors que la gamme des poids moléculaires des composés de ces fractions est très large (Planche $\mathrm{I}$ ).

\section{TABLEAU I}

Teneur en protéines et en sulfates des différentes fractions

Les résultats sont exprimés en pourcentage de poids sec. En ce qui concerne les protéines le dosage a été effectué sans hydrolyse préalable (protéines solubles) ou après hydrolyse par $\mathrm{NaOH} 2 \mathrm{~N}$ (à $100^{\circ} \mathrm{C}$ pendant Io minutes) (protéines totales) (LowRy et al., 195I); la référence utilisée a été la sérum-albumine bovine (fraction V) traitée dans les mêmes conditions. La précision des mesures des concentrations en protéines n'est pas indiquée puisque celles-ci n'ont qu'une valeur indicative (voir texte).

\begin{tabular}{c|c|c|c|c}
\hline & FI & FM & FEi & FEe \\
\hline $\begin{array}{c}\text { Protéines } \\
\text { solubles }\end{array}$ & 22 & $0-5$ & 26 & $0-5$ \\
\hline $\begin{array}{c}\text { Protéines } \\
\text { totales }\end{array}$ & 22 & 46 & 26 & 34 \\
\hline Sulfates & $4,4 \pm 1,2^{*}$ & $0,5 \pm 0,5$ & $2,8 \pm 0,6$ & $1,1 \pm 1,0$ \\
\hline$\ldots$
\end{tabular}

* Intervalle de confiance $(p=0,05 ; n=7)$.

Nous ne retiendrons que la nette différence de solubilité dans la soude $\frac{\mathrm{N}}{\mathrm{I} o}$ contenant 2 p. Ioo de $\mathrm{Na}_{2} \mathrm{CO}_{3}$ : les protéines des fractions FI et $\mathrm{FEi}$ peuvent être considérées comme solubles alors que celles des deux autres fractions sont nettement insolubles. 


\section{Composition en acides aminés}

I,es compositions en acides aminés des différentes fractions sont indiquées dans le tableau 2.

TABLEAU 2

Composition en acides aminés des différentes fractions

(exprimée en moles pour cent moles d'acides aminés totaux)

Il a été tenu compte des cinétiques de libération et de dégradation des divers acides aminés en fonction de la durée de l'hydrolyse

$$
(\varepsilon=\text { traces }) \text {. }
$$

\begin{tabular}{|c|c|c|c|c|}
\hline & FI & $\mathrm{FM}$ & FEi & $\mathrm{FEe}$ \\
\hline As $x$ & 5,9 & 9,5 & 6,6 & 9,1 \\
\hline Thr & 25,6 & 12,3 & 27,5 & 14,3 \\
\hline Ser & 14,0 & 7,7 & 19,0 & 11,8 \\
\hline Glx & 7,2 & 9,2 & 6,2 & 9,9 \\
\hline Pro & 12,3 & 9,9 & 7,8 & 8,1 \\
\hline Gly & 5,7 & 7,0 & 5,7 & 5,7 \\
\hline Ala & 2,7 & 4,8 & 3,1 & 5,0 \\
\hline Val & 6,5 & 6,7 & 4,4 & 6,0 \\
\hline Cys & $\varepsilon$ & 5,9 & 3,9 & 4,1 \\
\hline Met & $\varepsilon$ & $\varepsilon$ & $\varepsilon$ & 0,7 \\
\hline Ile & 3,4 & 4,2 & 2,8 & 4,7 \\
\hline Leu & 3,5 & 5,7 & 3,2 & 6,4 \\
\hline Tyr & 2,6 & 4,2 & 2,2 & 2,4 \\
\hline Phe & 2,4 & 4,3 & 2,0 & 2,6 \\
\hline Lys & 4,1 & 4,6 & 2,6 & 3,6 \\
\hline $\mathrm{His}$ & 1,7 & 0,9 & 0,8 & 1,2 \\
\hline Arg & 2,3 & 3,2 & 2,1 & 4,4 \\
\hline Total & 100 & 100 & 100 & 100 \\
\hline
\end{tabular}

La somme des concentrations de divers acides aminés regroupés selon les critères indiqués ci-après apparaissent dans la figure 2 . Les acides aminés retrouvés sous forme de résidus acides après hydrolyse (AsX et G1X) sont plus abondants dans les fractions FM et FEe, alors que les acides aminés possèdant une fonction alcool, sont nettement plus abondants dans les fractions FI et FEi. Bien que les différences soient moins accentuées que précédemment, on peut noter par ailleurs que les acides aminés possèdant une chaîne latérale hydrophobe (proline, alanine, valine, méthionine, isoleucine, leucine et phénylalanine) se trouvent en quantité plus grande dans les fractions classées insolubles lors du dosage des protéines (FM et FEe). Notons enfin que c'est la fraction moyenne qui possède le plus de cystéine, acide aminé pratiquement absent de la fraction interne. 


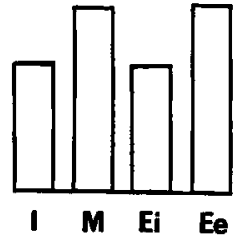

A

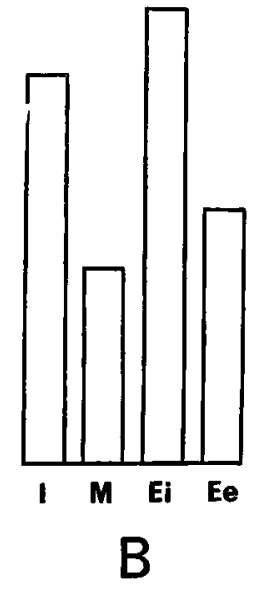

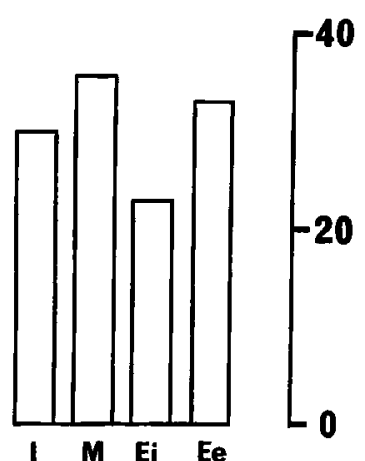

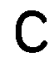

Fig. 2. - Comparaison des teneurs en divers groupes d'acides aminés

A : acides aminés apparaissant après hydrolyse sous forme de diacides. B : acides aminés à fonction alcool. $\mathrm{C}$ : acides aminés à chaîne latérale hydrophobe. I, M, Ei et Ee désignent les fractions FI, FM, FEi et FEe. Les résultats sont exprimés en moles pour cent moles d'acides aminés totaux. Les fractions FI et FEi possèdent nettement plus d'acides aminés à fonction alcool que les deux autres fractions qui sont par contre, plus riches en acides aspartique et glutamique (après hydrolyse) et en acides aminés à chaîne latérale hydrophobe.

\section{Teneur en ions sulfates}

Les résultats qui apparaissent dans le bas du tableau I montrent que les fractions FI et FEi sont assez riches en ions sulfates (4,4 et $2,8 \mathrm{p}$. Ioo du poids sec) alors que les deux autres fractions en contiennent très peu ( $I, I$ et 0,5 p. IOo).

\section{DISCUSSION}

Les quatre fractions que nous avons isolées à partir des gangues peuvent se classer en deux catégories. La première catégorie comprend les fractions FI et FEi. Elles possèdent des constituants à poids moléculaires inférieur ou égal à roo ooo daltons qui réagissent comme des composés solubles dans le dosage par la technique de Lowry et sont riches en sérine, thréonine et en ions sulfates. La deuxième catégorie comprend les fractions FM et FEe. Elles sont composées de constituants dont le poids moléculaire est supérieur ou nettement supérieur à Ioo 000 daltons qui réagissent comme des composés insolubles dans le dosage par la technique de Lowry et sont plus riches en acides aspartique et glutamique après hydrolyse ; ils sont par contre très peu sulfatés.

En ce qui concerne la taille des constituants des fractions moyenne (FM) et surtout externe (FEe) des gangues ovulaires de pleurodèle, nos résultats sont à rapprocher de ceux d'OLIPHANT et HEDRICK (I97I) obtenus sur la gangue externe des œufs de Xenope. Ces auteurs trouvent à ce niveau un constituant dont le poids moléculaire est voisin de I,5 million de daltons. Bien qu'insoluble dans l'eau (rappelons que les œufs de Pleurodèles entourés de leurs gangues sont pondus naturellement dans l'eau), la fraction externe y subit une imbibition qui se traduit par un important gonflement ce qui n'est pas le cas de la fraction moyenne. Il est probable que les 
causes de l'insolubilité de ces deux fractions ne soient pas les mêmes; une étude précise de la solubilisation de ces deux fractions sous 1 'influence de divers agents physiques et chimiques est actuellement en cours.

La contamination des différentes fractions entre elles peut avoir deux origines : elle peut être expérimentale ou physiologique. De faibles quantités de produits peuvent être mélangées accidentellement par la technique de fractionnement qui est essentiellement manuelle. Mais il est possible de concevoir l'existence d'une contamination physiologique, c'est-à-dire naturelle, se traduisant par une migration de composés à travers les diverses gangues. D'après la planche $I$, nous voyons que ce sont surtout les petites molécules qui sont communes à toutes les fractions. Ce problème important pour l'étude de la fécondation sera repris par immunofluorescence effectuée sur des coupes d'œufs enveloppés de leurs gangues.

Nos résultats concernant la teneur en protéines totales des différentes fractions (22 à 46 p. Ioo selon les fractions), sont comparables à ceux que LEE (I967) a obtenu sur l'ensemble des gangues ovulaires de la grenotille (29 à $4 \mathrm{I} \mathrm{p.} \mathrm{I00);} \mathrm{ils} \mathrm{sont} \mathrm{par}$ contre légèrement plus faibles que ceux de MiNGantr et d'ANNA (I957 et r958) qui indiquent 52,5 p. roo de protéines dans l'ensemble des gangues ovulaires de Triturus cristatus et 54,4 p. roo dans celles de Discoglossus pictus.

Quant à la composition en acides aminés, nos résultats divergent notablement de deux de Fol,kes et al., I950 qui ont trouvé dans la gangue totale des œufs de grenouille une proportion importante de glycine (environ 21 moles p. Ioo moles d'acides aminés totaux) et relativement faible d'acides aminés possédant une fonction alcool (Ser + Thr $=$ I2 moles $p$. Ioo moles d'acides aminés totaux). La forte proportion de sérine et de thréonine dans nos quatre fractions montre qu'elles diffèrent beaucoup des sécrétions de l'oviducte des oiseaux (OSUGA et FEENEY, I968 ; FOTHERGILL, et FothergILL, I970). Les fractions FI et FEi possèdent pourtant bien les caractéristiques des sécrétions muqueuses mentionnées par BUDDECKE (I966) : teneurs élevées en thréonine, sérine et proline, faibles en acides aminés aromatiques ou soufrés. C'est la fraction FM qui possède la plus grande teneur en cystéine expliquant au moins partiellement ses caractéristiques physiques.

On retrouve donc pour les composants protidiques une hétérogénéité analogue à celle observée pour les composants glucidiques (JEGO, I974). Le matériel entourant l'œuf ne doit en aucun cas être considéré comme une entité mais comme une succession de couches chimiquement distinctes. S'il est possible de classer ces gangues en deux catégories à cause de certaines ressemblances physiques et chimiques, les gangues d'une même catégorie sont toujours séparées anatomiquement par celle de l'autre catégorie. Il est pratiquement certain que les rôles joués par les gangues dans la fécondation sont différents; les présents résultats permettront peut être d'en aborder l'étude dans de meilleures conditions.

Reçu pour publication en mai 1975.

\section{REMERCIEMENTS}

Que Mmes C. Calvez, M. Mathelier, A.-M. Touzalin et M. D. Blanchet soient remerciés pour leur participation technique à l'élaboration de ce travail.

Travail accompli dans le cadre de l'A.T.P. du C.N.R.S. " Mécanisme d'action des hormones "(Contrat no I349). 


\section{SUMMARY}

\section{PROTEIN ANAIYSIS IN THE EGG CAPSULES OF PLEURODELES WALTLII MICHAH}

Four fractions (FI, FM, FEi and FEe) have been mechanically isolated from egg jelly coat of Pleurodeles waltlii мICHAH.

All the fractions are rich in serine and threonine, however, their proteins are very different ; fractions can be ordered into two groups :

I. FI and FEi (corresponding to the inner layer and the inner part of the external layer) have sulfated components which molecular weight is lower or equal to roo ooo daltons; they are water soluble or readily soluble in dilute alkali and particulary rich in serine and threonine.

2. FM and FEe (corresponding to the middle layer and the external part of the external layer) have components which molecular weight are heavier than Ioo ooo daltons; they are insoluble in water or dilute alkali, rich in glutamic and aspartic acids and in amino acids containing non polar lateral chains.

\section{RÉFÉRENCES BIBLIOGRAPHIQUES}

Aplington H. W., 1957. The insemination of body cavity and oviducal eggs of Amphibia. Ohio. J. Sci., 57, 91-99.

Barbieri F. D., Oterino J. M., 1972. A study of the diffusible factor released by the jelly of the egg of the toad, Bufo arenarum. Develop. growth Different., 14, I07-II 7 .

BARbieri F. D., Raisman J. S., I 969 . Non gametic factors involved in the fertilization of bufo arenarum ovocytes. Fimbryologica, 10, 363-372.

Bataillon E., igrg. Analyse de l'activation par la technique des œufs nus et la polyspermie expérimentale chez les Batraciens. Ann. Sci. Nat. Zool., 10, I-38.

Borsseau C., I974. Étude ultrastructurale de l'oviducte du Triton Pleurodeles wallii michar. III. Action de l'œestradiol-i $\rangle$ sur la différenciation des cellules sécrétrices de l'oviducte moyen de la femelle immature. (Soumis pour publication).

Boisseau C., Jego P., Joly J., Picheral B., I974. Organisation et caractérisation histochimique des gangues ovulaires sécrétées par l'oviducte de Pleurodeles waltlii мıснан. (Amphibien, Urodèle, Salamandridé). C. R. Soc. Biol., 168 (sous presse).

Buddecke E., I966. In Glycoproteins. A. Gottschalk (Ed.) BBA Library Vol. 5, EIsevier, Amsterdam, p. 558 .

Dogson K. S., Price R. G., I962. A note on the determination of the ester sulphate content of sulfated polysaccharides. Biochem. J., 84, 106-1 10.

Elinson R. P., I97I. Fertilization of partially jellied and jellyless ovocytes of the frog Rana pipiens J. Exp. Zool, 176, p. 415-428.

Folkes B. F., Grant R. A., Jones J. K. N., I950. Frog-spawn mucin. J. Chem. Soc., 440, $2136-$ $2 \mathrm{I} 4 \mathrm{O}$.

Fothergill L. A., Fothergill J. L., r97o. Structural comparison of Ovalbumius from nine different species. Eur. J. Biochem., 17, 529-532.

Glick R. N., Shaver J. R., I963. The fertilizability of frog eggs from different levels of the female reproductive tract. Exptl. Cell. Res., 32, 615.6I8.

Good G. M., Daniel J. F., I943. Fertilization of coelomic eggs of triturus torosus. Univ. Calif. Publ. Zool., 51, I49-I58.

HJERTEN S., 1962. "Molecular Sieve "Chromatography on polyacrylamide gels, prepared according to a simplified method. Arch. Bioch. Biophys. Suppl., 1, 147-r 5r.

Hughes N., r957. An investigation into the fertilizability of coelomic and oviducal eggs of the newt triturus viridescens. $A$. S. B. Bull., 4, I3.

JEGo P., I974. Composition en glucides des différents segments de l'oviducte et des gangues ovulaires chez Pleurodeles waltii michar. (Amphibien, Urodele). Comp. Biochem. Physiol., 48 B, 435-446.

Jego P., Valotaire Y., Joly J., 1974. Effets du $\beta$-cestradiol sur l'activité de la thymidine kinase de l'oviducte du triton Pleurodeles waltlii міснан Gen. Comp. Endocrinol. (sous presse). 
Kambara J., 2953. Role of the jelly envelopes of toad eggs in fertilization. Ann. Zool. Jap., 26, 78-8I.

Katagiri C., I966. Fertilization of dejellied uterine toad eggs in various experimental conditions. Embryologica, 9, I 59-I69.

Katagiri C., I973. Chemical analysis of toad eggs-jelly in relation to its " sperm-capacitating " activity. Develop. Growth Different., 15, 81-92.

LEE P., I967. Studies of frog oviducal jelly secretion. I. Chemical analysis of secretory product. Exp. Zool., 166, 99-ro6.

Lowry O. H., Rosebrough N. J., FarR A. L., Randall R. J., I95I. Protein measurement with the folin phenol reagent. J. Biol. Chem., 193-265.

Minganti A., D'Anna T., I957. Ricerche Sulla mucina ovulare di Triton cristatus. La Ricerca Scientifica Anno., 27, 3052-3054.

Minganti A., D'Anna T., 1958. Sulla composizione della mucina ovulare di Discoglossus Pictus. La Ricerca Scientifica Anno., 28, 2090-2093.

Nadamitsu S., I957. Fertilization of coelomic and oviducal eggs of Triturus pyrrogaster. J. Sci. Hiroshima. Univ. Ser. B, 17, 51-53.

Nevilie D. M., I97I. Molecular weight determination of protein-dodecyl sulfate complexes by gel electrophoresis in a discontinuous buffer system. J. Biol. Chem., 246, 6328-6334.

OLIPHANT G., HEDRICK J. L., I97I. Isolation and physicochemical characterization of a sperm capacitation factor from the jelly coat of Xenopus laevis eggs. Fed. Proc., 30,1280 (abst.).

Osuka D. T., Feeney R. E, 1968. Biochemistry of the egg white proteins of the ratite group. Arch. Bioch. Bioph., 124, 560-574.

Picard J., Gardais A. P., Vedel M., i973. Glycoprotéines sulfatés des membranes de l'ceuf de poule et de l'oviducte. Isolement et caractérisation de glycopeptides sulfates. Biochem. Biophys. Acta, 320, 427-44I.

Shaver J. R., Barch S. H., ig60. Experimental studies on the role of jelly coat material in fertilization in the frog. Acta. Embryol. Morphol. Exp., 3, 180-189.

Shivers C. A., James J.-M., 1970. Capacitation of frog sperm. Nature, 227, I83-184.

Subtelny S., Bradt C., I96r. Transplantations of blastula nuclei into activated eggs from the body cavity and from the uterus of Rana pipiens. II. Development of the recipient body cavity eggs. Develop. Biol., 3, 96-I I4.

Tchou-Su, WANG Y. L., 1956. Études expérimentales sur le rôle du mucus des oviductes dans la fécondation chez le Crapaud et la considération générale sur le mécanisme de la pénétration spermatique. Acta. Exp. Biol. Sinica, 5, 75-I22.

Wolf D. P., Hedrick J. L., I97I. A molecular approach to fertilization. III. Development of a bioassay for sperm capacitation. Dev. Biol., 25, 360-376.

\title{
PLANCHE I
}

\author{
Photographies des gels colorés par l'amido-schwartz \\ après migration des différentes fractions \\ (dépôts de Ioo $\mu \mathrm{g}$ de protéines)
}

En dessous de chaque photographie est reporté le graphe obtenu après lecture au densitomètre des négatifs des photographies correspondantes. Ainsi qu'une échelle des poids moléculaires (en milliers de daltons) réalisée en se référant à la migration de protéines connues ; cette échelle s'étend du début du gel de diffusion au front de migration. L,es fractions se répartissent nettement en deux catégories selon le poids moléculaire de leurs constituants : inférieur ou égal à I0o ooo daltons pour FI et FEi ; supérieur ou nettement supérieur à roo ooo daltons pour FM et FEe. 

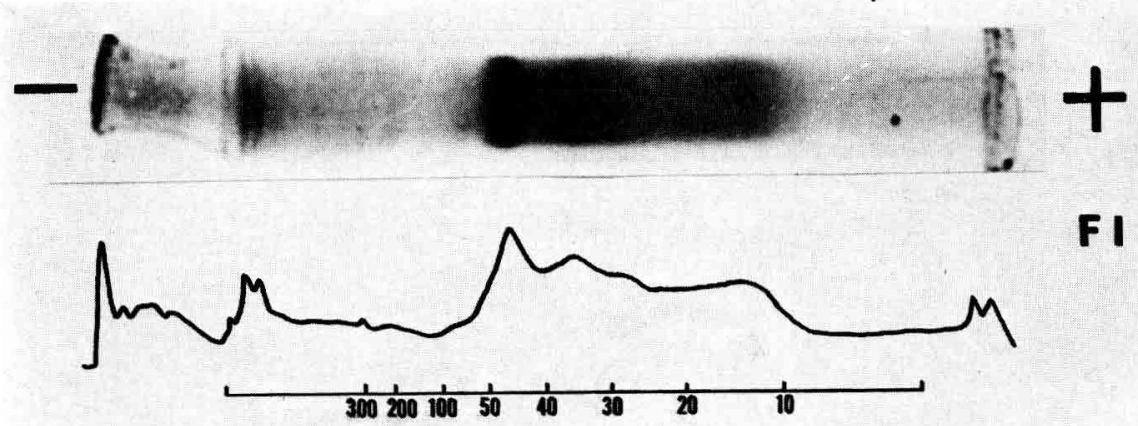

F I
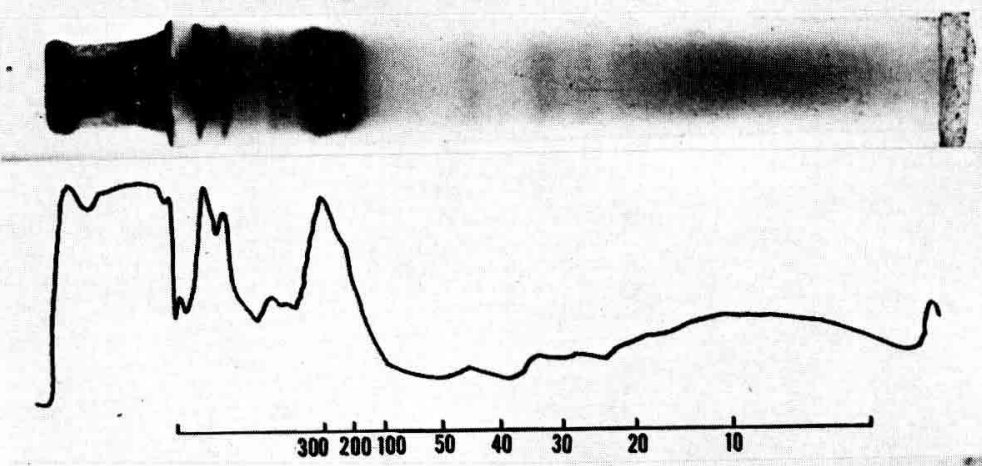

F M
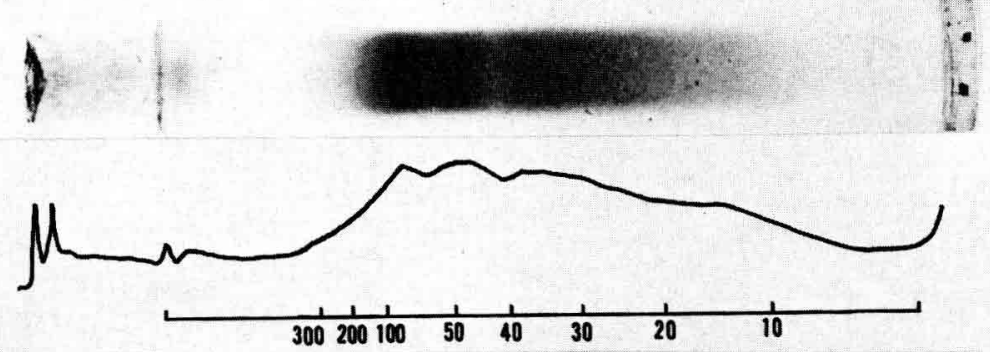

FE i

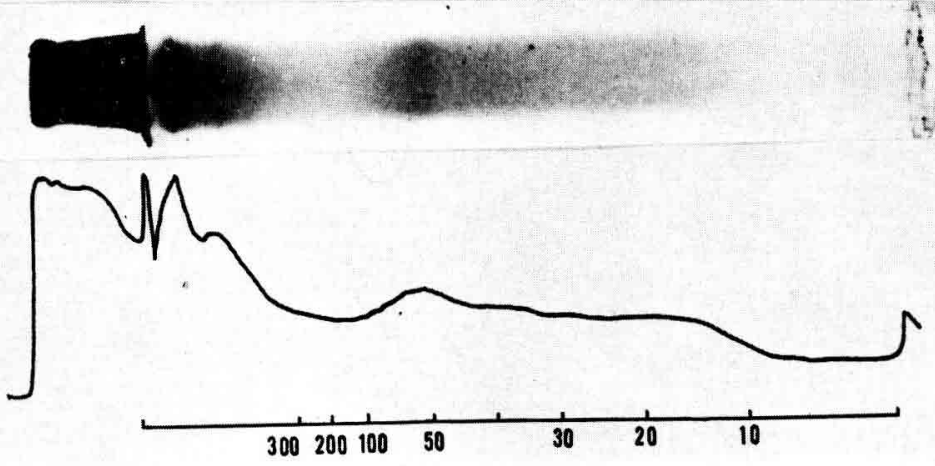

FEe

P. JEGO 